\title{
Transit jüdischer Flüchtlinge durch Spanien ab 1940
}

Spaniens Haltung gegenüber Flüchtlingen - ob Juden oder nicht - war während des Zweiten Weltkriegs von Bereitschaft zur Gewährung von Transitvisa geprägt, während zugleich versucht wurde, einen dauernden Aufenthalt von Flüchtlingen zu verhindern. So klar diese Grundlinie der spanischen Flüchtlingspolitik bestimmt werden kann, so verwirrend war sie im Detail. Beginnen wir mit den juristischen Regelungen.

Grundlegend ist der Erlass vom 11. Mai 1939. Er behandelte allgemein die Ein- und Ausreise. Was fehlte, war eine gesonderte Regelung für Transitreisende. Spanischen Bürgern wurde die Einreise nur gestattet, wenn es keine Zweifel an ihrer Unterstützung des franquistischen «Movimiento» gab. Damit blieben exilierte Republikaner ausgeschlossen. Besondere Einschränkungen für Juden spanischer Staatsangehörigkeit gab es nicht. Anders sah es bei den Bestimmungen zur Einreise von Ausländern aus. Das Visum sollte folgenden Antragstellern verweigert werden:

- die gegen die «nationale» Bewegung gearbeitet hatten;

- die Geschäftsbeziehungen mit der Republik gepflegt hatten;

- Freimaurern;

- die Leitungsposten in Betrieben im republikanischen Gebiet oder mit «deutlich jüdischem Charakter» innegehabt hatten;

- Juden, es sei denn, dass sie in besonders freundschaftlichen Beziehungen zu Spanien standen und erwiesenermaßen die «Nationale Bewegung» unterstuitzt hatten.

Wie festgestellt werden sollte, wer Jude (oder Freimaurer) war, blieb offen; das beigefügte Formular für den Visumantrag sah keine entsprechende Frage vor. Bei deutschen Juden war jedoch durch das kurz zuvor eingeführte «J» in den Pässen auch für Spanien die Diskriminierung möglich. ${ }^{1}$ Spanien schottete

1 AMAE, Handbibliothek der Archivverwaltung, Erlasssammlung (ohne Signatur): Servicio Nacional de Política y Tratados, Orden Circular Nr. 90, Burgos, 11. Mai 1939. Marquina/Ospina, S. 146, interpretieren den Erlass so, dass gegnerisch gesonnenen Juden die Einreise verweigert werden sollte. Der Wortlaut des Erlasses geht aber darüber hinaus, indem von den ausländischen Juden positiv eine Unterstützung der «Nationalen Bewegung» gefordert wurde. 
sich damit gegen ausländische Juden ab, ohne die Entscheidung mit Argumenten wie dem Hinweis auf die schwierige Wirtschaftslage zu kaschieren. Für die spanischen Bürger gab es jedoch keine Unterscheidung nach «Rasse» oder Religion. Dies macht deutlich, dass Juden nicht als die gefährlichsten Feinde des Franco-Regimes angesehen wurden. An der Spitze dieser Rangliste standen die politischen Gegner; ihnen wurde die Einreise verwehrt, egal ob sie spanische Bürger oder Ausländer waren. Es folgten die Freimaurer, die ausdrücklich nur bei den Ausländern erwähnt wurden, die aber wohl stillschweigend zu den politisch Unzuverlässigen gerechnet wurden. Ausländische Juden schließlich waren nicht so grundsätzlich von der Einreise ausgeschlossen, wie die beiden anderen Gruppen. Einschränkend gilt aber, dass schon die leitende Tätigkeit in «jüdischen» Betrieben ein Hinderungsgrund für die Einreise von Ausländern war; in diesem Punkt traf das Dekret Juden schwerer als Freimaurer.

Es ist nicht klar, wie lange die Verordnung galt. Marquina/Ospina schreiben, sie sei mit dem Runderlass vom 1. Mai 1940 aufgehoben worden. ${ }^{2}$ Schon zuvor, am 2. Januar 1940, war ein auf den 4. September 1939 datierter Erlass an die spanischen Vertretungen im Ausland verschickt worden. Er regelte die Einreise von Spaniern und Ausländern und enthielt nicht mehr die Einschränkungen für Freimaurer und Juden. ${ }^{3}$ Damit schien der Erlass vom 11. Mai 1939 aufgehoben zu sein. Zweifel an dieser Sichtweise ließ aber der Erlass vom 1. Mai 1940 aufkommen. Er nahm sowohl auf die Regelung vom 11. Mai wie auf die vom 4. September 1939 als weiterhin geltende Bestimmungen Bezug, die nun für die Einreise von Ausländern modifiziert werden sollten, was zugleich hieß, dass sie nicht gänzlich aufgehoben wurden. Auch in diesem Erlass fehlten Einschränkungen für Juden, doch wurde nicht ausgeführt, welche der Bestimmungen vom 11. Mai 1939 weiter in Kraft bleiben sollten. ${ }^{4}$ Ein Schreiben des Außenministeriums vom Dezember 1942 bezeichnete den Erlass vom 11. Mai 1939 zur Gänze als noch gültig. ${ }^{5}$ Es scheint daher so zu sein, dass der Erlass vom Mai 1939 die ganzen Jahre über weiter galt.

2 Marquina/Ospina, S. 146. Sie schreiben dort auch, dass der genaue Text des Runderlasses 152 vom 1. Mai 1940 nicht auffindbar sei. Er befindet sich in: AMAE, Handbibliothek der Archivverwaltung, Eriasssammlung (ohne Signatur)

${ }^{3}$ AMAE, Handbibliothek der Archivverwaltung, Erlasssammlung (ohne Signatur): Dirección General de Política y Tratados, Sección de Pasaportes, Madrid: Erlass 130, 2. Jan. 1940.

4 Ebd.: Erlass 152, 1. Mai 1940.

5 AMAE, R 2153/41: Außenministerium Madrid an Spanisches Generalkonsulat Brüssel, 23. Dez. 1942. 


\section{Deutsche und französische Auswanderungsvorschriften für Juden}

Bevor wir uns mit der weiteren Entwicklung der spanischen Flüchtlingspolitik beschäftigen, sollen die deutschen und französischen Auswanderungsvorschriften vorgestellt werden, konditionierten sie doch die spanische Politik in starkem Maße, wenn auch erst seit der Besetzung Frankreichs, denn erst mit ihr setzte ein starker Zustrom von Flüchtlingen nach und durch Spanien ein. Das deutsche Besatzungsgebiet grenzte nur am westlichen Rand der Pyrenäen an Spanien, schloss aber den bedeutenden Bahn- und Straßenübergang von Hendaye (Frankreich) nach Irun ein. Der größte Teil der spanischen Nordgrenze stand unter der Kontrolle der Vichy-Regierung, auf deren Gebiet die deutschen Ausreisebestimmungen bis Ende 1942 nicht galten. Besonders wichtig war in diesem Bereich der Kontrollpunkt Cerbère (Frankreich) Portbou (Spanien) am Mittelmeer.

Prinzipiell gestattete bis 1941 die deutsche Seite die Auswanderung von Juden. Enteignungsähnliche Ausreiseabgaben und der Mangel an aufnahmewilligen Ländern gestalteten die Emigration aber schwierig. Im Frühjahr 1941 wurde sie für das besetzte Frankreich auch offiziell gestoppt. Am 20. Mai übermittelte das Reichssicherheitshauptamt (RSHA) dem Beauftragten des Chefs der Sicherheitspolizei und des SD (BdS) für Belgien und Frankreich eine «Mitteilung» von Göring. Der Reichsmarschall forderte das Ende der Auswanderung von Juden aus Frankreich und Belgien, um die Ausreisemöglichkeiten für Juden aus Deutschland zu verbessern. Es war also kein prinzipielles Ende jüdischer Emigration beabsichtigt, sondern die Bevorzugung der Ausreise aus dem Reichsgebiet, mit dem Ziel, dieses schneller «judenfrei» zu machen. Aber auch für Frankreich bedeutete diese Anordnung noch kein völliges Ende der Auswanderungsmöglichkeit, denn die deutsche Seite hatte nur im besetzten Teil Frankreichs die uneingeschränkte Macht. ${ }^{6}$ Dies zeigte auch die Formulierung im Telegramm aus Paris an die deutsche

6 PA AA, R 100869: RSHA Berlin, Schellenberg, an alle Staatspolizeistellen und an den Beauftragten des Chefs der Sicherheitspolizei und des SD für Belgien und Frankreich, 20. Mai 1941. Dieses Dokument, das für die Rekonstruktion des Entschlusses zur Ermordung aller Juden wichtig ist, wird von Hilberg, Vernichtung, S. 419, nicht korrekt wiedergegeben. Seine Zusammenfassung lautet: «Am 20. Mai 1941 teilte ein GestapoBeamter des RSHA, Walter Schellenberg, dem Militärbefehlshaber in Frankreich mit, die Auswanderung von Juden aus seinem Verwaltungsgebiet sei zu verhindern, weil nur beschränkte Transportmittel zur Verfügung stünden und zudem <die Endlösung der Judenfrage > nunmehr in Sicht sei.» Hier fehlt der entscheidende Passus, dass die Auswanderung aus Frankreich und Belgien nur deswegen zu verhindern sei, weil sonst die Auswanderung aus Deutschland stocken würde. Endlösung der «Judenfrage» ist hier nicht, wie es bei Hilberg den Anschein hat, als Ermordung zu lesen. Hätte Göring am 20. Mai 1941 unter «Endlösung» die Ermordung möglichst vieler Juden verstanden, hätte er jegliche Auswanderung stoppen müssen. 
Amtsstelle in Bordeaux, mit dem der Erlass übermittelt wurde. Dort hieß es, dass «eine Auswanderung von Juden aus dem besetzten Westgebiet gleich wie auch nach Möglichkeit [Hervorh. des Verf.] aus dem unbesetzten Frankreich zu unterbinden ist.»»?

Das Auswanderungsverbot für Juden aus dem gesamten deutsch besetzten Europa folgte - auf Anordnung von Himmler - am 23. Oktober 1941. Der BdS in Belgien und Frankreich - Himmlers dortiger Repräsentant - wurde aufgefordert, «die in Frage kommenden innerdeutschen Behörden des dortigen Dienstbereiches von dieser Anordnung zu unterrichten.» ${ }^{8}$ Dies hieß, dass nicht ausdrücklich versucht wurde, das Auswanderungsverbot auch im unbesetzten Teil Frankreichs durchzusetzen. Und selbst im besetzten Teil des Landes dauerte es bis zum 4. Februar 1942, bis der Militärbefehlshaber den Erlass in seiner Verwaltung weitergab. ${ }^{9}$

Vichy-Frankreich schloss sich vorerst dem deutschen Kurswechsel nicht an. Hier blieb grundsätzlich die Auswanderung noch möglich, ja offizielle Politik war sogar deren Förderung, doch sah die Praxis oft anders aus. In der ersten Zeit nach der französischen Kapitulation mussten alle Ausreiseanträge nach Vichy übermittelt werden, von wo sie an die deutsch-französische Waffenstillstandskommission in Wiesbaden weitergeleitet wurden, um die Zustimmung der deutschen Seite einzuholen. 1940 war Letzteres noch kein prinzipielles Problem. Schwierigkeiten gab es eher auf französischer Seite. Zeitzeugen berichten, dass sie von ihr keine Antwort auf derartige Anträge erhielten; wahrscheinlich lag es daran, dass die Verwaltung in Vichy erst aufgebaut werden musste. Nachdem dieses gelungen war, erhielt die französische Regierung die alleinige Kompetenz in der Auswanderungsfrage zurück. Was dies praktisch bedeutete, wird in einer Schilderung aus dem Oktober 1941 deutlich. Das französische Ausreisevisum musste beim Präfekten des Departments beantragt werden. Manchmal verlangte dieser ein Führungszeugnis, was eine Nachfrage beim Polizeikommissariat erforderte. Für staatenlose Juden oder solche aus kriegführenden Staaten musste von der Präfektur beim Innenministerium nachgefragt werden. Wer per Schiff ausreisen wollte, was nur in Marseille möglich war, musste sich auch an die Präfektur des dortigen Departments wenden, das die Schiffsplätze verwaltete. Bei der Banque de France mussten die Dollars beantragt werden, um damit die Fahrkarten zu kaufen. In Lagern internierte Juden - dazu gehörten viele, die nicht

${ }^{7}$ Internationaler Militärgerichtshof, Bd. 7, S. 37, Dok. RF-1202: Telegramm Paris [nicht näher spezifiziert] an Amtsstelle Bordeaux [nicht näher spezifiziert], 26. Juli 1941.

${ }^{8}$ Zitiert nach der ungekürzten Wiedergabe des Schreibens an den BdS in Belgien und Frankreich in: Longerich, S. 82.

${ }^{9}$ Marrus/Paxton, S. 231. 
die französische Staatsbürgerschaft besaßen - konnten dieses alles nur regeln, wenn sie freigelassen oder in das Transitlager Milles bei Aix-en-Provence überstellt wurden. Wer nicht im Lager interniert war, benötigte Passierscheine, um zu den zuständigen Behörden zu gelangen. Zudem hatten alle Dokumente nur begrenzte Gültigkeitsdauer; wer nicht alles innerhalb dieser Fristen erledigen konnte, musste das entsprechende Dokument erneuern lassen oder von vorne anfangen.

Am 20. Juli 1942 stoppte der französische Innenminister die Gewährung von Ausreisevisa an Juden, mit Ausnahme der Belgier, Holländer und Luxemburger, um über genügend ausländische Juden zu verfügen, die der deutschen Seite zur Deportation überstellt werden konnten. ${ }^{10}$ Mit Hilfe der jüdischen Flüchtlingshilfeorganisation HICEM konnten in der zweiten Jahreshälfte 1942 nur noch 600 Juden aus Vichy-Frankreich auswandern, gegenüber 2.000 in der ersten Jahreshälfte und 3.000 im Vorjahr. Am 8. November 1942 - noch vor dem Einmarsch der deutschen Truppen in die unbesetzte Zone also - wurde unter deutschem Druck die legale Emigration von Juden aus Vichy-Frankreich gänzlich unterbunden. Aber auch zuvor war die Zahl der Auswanderer unter den Aufnahmekapazitäten der Zielländer geblieben. ${ }^{11}$

Es bleibt als Fazit, dass aus dem deutsch besetzten Teil Frankreichs bis zum Frühjahr 1941 und aus dem übrigen Landesteil bis Sommer 1942 die Auswanderung von Juden in größerem Umfang möglich war. Danach gab es für die meisten Juden nur noch die Flucht über die Grenze.

\section{Spanische Vorschriften für die legale Ein- und Durchreise und} ihre Anwendung ab Juni 1940

Wie reagierte Spanien auf Versuche von Juden, einzureisen oder - was häufiger war - das Land im Transit zu passieren? Für die Einreise hatte es am 1. Mai 1940 - also wenige Tage vor dem deutschen Angriff auf Frankreich per Erlass eine Modifikation der Verordnung vom 11. Mai 1939 gegeben. Neu war die Regelung, wer über die Visa zu entscheiden hatte. Die Konsulate durften nur Empfehlungen abgeben und mussten alle Anträge von Ausländern an

${ }^{10}$ Wir haben keine Erklärung dafür gefunden, dass die Juden aus den BeNeLux-Staaten von dieser Bestimmung ausgenommen wurden.

"Schilderung der Situation in Vichy-Frankreich nach: Marrus/Paxton, S. 154-156, 230-232. Unter Berufung auf Sekundärliteratur schreiben die Autoren aber zugleich und ohne nähere Erläuterung, HICEM habe zwischen Juni 1940 und März 1943 24.000 Juden bei der legalen Auswanderung unterstützt. Diese Zahl erscheint uns überhöht. 
die Sicherheitspolizei (Dirección General de Seguridad) nach Madrid senden. Dort wurde die definitive Entscheidung getroffen. Nur in äußerst dringenden und besonders begründeten Fällen durften die Konsuln ohne diese Anfrage Einreisevisa ausstellen, mussten dann aber unverzüglich das AuBenministerium in Madrid davon in Kenntnis setzen, das wiederum die DGS informierte. $^{12}$

Transitvisa hingegen konnte ohne vorherige Nachfrage in Madrid jeder Antragsteller, der das portugiesische Einreisevisum vorwies, von den spanischen Konsulaten erhalten. ${ }^{13}$ Diese Regelung kam den Flüchtlingen entgegen, denn der Transit war ja das, was so gut wie alle von ihnen anstrebten. In der Regel legte Spanien der Durchreise von Flüchtlingen - fast immer nach Portugal, von wo die wenigen verbliebenen Atlantikrouten abgingen - keine Hindernisse in den Weg. ${ }^{14}$ In dem von einer Diktatur, die mit den Nationalsozialisten sympathisierte, regierten und vom Bürgerkrieg verwüsteten Land wollte kaum einer von ihnen länger als nötig bleiben.

Während des französischen Zusammenbruchs im Juni 1940 war der bevorzugte Grenzübergang für die vielen Flüchtlinge, die das Land nach Süden verlassen wollten, Hendaye am Golf von Biscaya. Die spanischen Grenzbehörden im gegenüberliegenden Irun ließen alle Flüchtlinge mit portugiesischen Visa passieren; da der portugiesische Konsul in Bordeaux, Sousa Mendes, sie entgegen den Vorschriften seiner Regierung reichlich ausstellte, waren es nicht wenige. ${ }^{15}$ Der spanische Konsul in der französischen Grenz-

${ }^{12}$ Wortlaut des Erlasses in: Erlasssammlung des Spanischen Außenministeriums in der Handbibliothek des AMAE. Marquina/Ospina, S. 146, schreiben noch, der Erlass sei nicht auffindbar gewesen. Aus der Praxis in den folgenden Monaten schließen sie, dass der Erlass die Konsulate ermächtigt habe, nach eingehender Prüfung selber Transitvisa auszustellen. Dies trifft nicht zu.

${ }^{13}$ So ausdrücklich in: AGA, AAEE, 11767: Spanischer Konsul Hendaye an Spanische Botschaft in Frankreich, 15. Juli 1940.

14 Vgl. als ein besonders markantes Beispiel: Archivo de la Presidencia del Gobierno, Madrid: Sección Presidencia, Serie Secretaría Ministro Subsecretario, Legajo 8: Subsecretario [de Presidencia del Gobierno] an die Minister für Äußeres und Inneres, 21. Nov. 1941: Der Staatschef habe angeordnet, dass die französischen Bürger, die aus Frankreich wegen der Anordnungen der französischen Regierung gegen Freimaurer ausgewiesen wurden, nicht dauerhaft in Spanien bleiben dürfen. Dies schließe nicht aus, dass ihnen Transitvisa durch Spanien gewährt werden. Wer auch nur oberflächlich über Francos Abneigung gegenüber den Freimaurern informiert ist, erkennt das AuBergewöhnliche an dieser Entscheidung.

$15 \mathrm{Vgl}$. Afonso, Rui: Injustiça. O caso Sousa Mendes. Lisboa 1990. Der damalige Botschafter Portugals in Madrid, Pereira, schrieb dreißig Jahre später in seinen Memoiren, dass er am 20. Juni 1940 an die Pyrenäengrenze eilte und dort bei widerstrebenden spanischen Grenzbehörden die Einreise von Flüchtlingen mit portugiesischen Visa durchsetzte. Vgl. Pereira, Pedro Theotónio: Memórias. Postos em que servi e algumas recordações pessoais. 2 Bde. Lisboa 1972/3, Band 2, S. 220 . Ob diese Schilderung zutrifft, muss offen bleiben. Die Darstellungen zum Fall Sousa Mendes (Afonso; 
stadt Hendaye, Navarro Guimbao, schätzte, dass er innerhalb von acht Tagen etwa 2.000 Transitvisa ausgestellt habe. Dennoch wurden viele Flüchtlinge von der spanischen Grenzpolizei zurückgewiesen, da sie kein portugiesisches Visum besaßen. ${ }^{16}$ Navarro zufolge befanden sich insbesondere Juden ohne französische Staatsangehörigkeit in dieser Situation, während Besitzer französischer Pässe durchweg alle nötigen Visa vorweisen konnten. Er schreibt, dass die spanischen Kontrollen strikt waren, denn die Einreise von Juden nach Spanien sei nicht erwünscht gewesen. Auch Bestechungsversuche hätten an dieser Haltung nichts geändert. ${ }^{17}$ Es lässt sich nicht sagen, in welchem Umfang diese Schilderung zutrifft. Augenzeugenberichte und Sekundärliteratur stimmen darin überein, dass eine in die Tausende gehende Zahl von Flüchtlingen, darunter viele Juden, während des französischen Zusammenbruchs die spanische Grenze mit dem Ziel der Durchreise nach Portugal überschreiten konnte. Es scheint so gewesen zu sein, dass ihre Zahl deutlich über der der von Spanien zurückgewiesenen Flüchtlinge lag.

Am 27. Juni 1940 erreichten die deutschen Truppen die spanisch-französische Grenze in Hendaye. Sowohl die neuen Besatzungstruppen als auch Spanien schlossen nun die Grenze; Spanien bereitete strengere Kontrollen vor, mit der die Einreise von Republikanern verhindert werden sollte. Mittlerweile hatte sich aufgrund der deutschen Besetzung der französischen Atlantikküste der Flüchtlingsstrom von Hendaye/Irun am Westrand der Pyrenäen nach Cerbère/Port Bou am Mittelmeer verlagert. Anfang September 1940 wurde dieser Grenzkontrollpunkt wieder geöffnet. ${ }^{18}$

In den folgenden Monaten war die spanische Transitpolitik sehr wechselhaft. Für September 1940 berichtet Zur Mühlen von absichtlich schleppender Abfertigung an der Grenze. ${ }^{19}$ Es folgten innerhalb von drei Monaten vier Erlasse zur Regelung des Transits. Der erste von ihnen, vom 8. Oktober, wird mit dem Besuch von Himmler in Madrid in Verbindung gebracht, doch ist dieser Zusammenhang unsicher, zumal der SS-Führer erst am 20. Oktober in Madrid eintraf. ${ }^{20}$ Der Erlass erschwerte die Vergabe von Transitvisa dra-

Wheeler, Douglas L.: And Who is My Neighbor? A World War II Hero of Conscience for Portugal. In: Revista de Estudos Judaicos, 1 (1995), S. 19-36, zeigen eher umgekehrt, dass Pereira die Anerkennung der vom Konsul erteilten Visa verhindern wollte.

${ }^{16}$ Afonso, S. 85f.

${ }^{17}$ AGA, AAEE, 11767: Spanischer Konsul Hendaye an Spanische Botschaft in Frankreich, 15. Juli 1940. Navarros Aussage muss nicht bedeuten, dass es keine Fälle erfolgreicher Bestechung gab.

${ }^{18}$ Avni, Spain, S. 73.

${ }^{19}$ Zur Mühlen, S. 86f., 90.

${ }^{20}$ Marquina/Ospina, S. 147f., stellen den Zusammenhang mit dem Himmler-Besuch her. Payne, S. 272f., argumentiert, dass der Aufenthalt nur kurz gewesen und i. w. touristischen Attraktionen wie einem Stierkampf und einer Jagd gegolten habe. Zumindest das erste Argument überzeugt nicht recht, dauerte der Besuch doch drei Tage. Preston, 
stisch. Auch sie bedurften ab sofort der vorherigen Genehmigung durch das Außenministerium. Die Konsulate wurden angehalten, den Namen des Antragstellers, seine Nationalität und das Alter telegrafisch zu übermitteln, begleitet von einer Mitteilung, ob der Pass noch gültig war, ob es ein NansenPass war sowie ob das Visum des Ziellandes und alle Transitvisa vorlagen. Sollte in Spanien ein Schiff bestiegen werden, war auch der Name des Schiffes anzugeben und der Besitz einer Fahrkarte für das Schiff nachzuweisen. Die Kosten der telegrafischen Anfrage in Madrid hatte der Antragsteller zu übernehmen; andernfalls erfolgte sie per Brief. Eine Antwort sollte - bei telegrafischer Anfrage! - innerhalb von drei bis sechs Wochen erfolgen.

Es gab jedoch, wie die Spanische Botschaft in Frankreich den Konsulaten mitteilte, eine wichtige Ausnahme von diesen Vorschriften. Sie ist in bisherigen Untersuchungen nicht berücksichtigt worden. Danach konnte wie bisher der Konsul Transitvisa ohne Rückfrage ausstellen, wenn die Antragsteller nicht Bürger kriegführender Staaten waren. Sie mussten nur über ein portugiesisches Einreisevisum verfügen, den Ort der Ein- und der Ausreise aus Spanien angeben und Garantien vorweisen, sprich: genügend Geld zur Finanzierung des Transits besitzen. Dies entsprach der bisherigen Regelung. ${ }^{21}$

In der Praxis hieß dies: staatenlose Juden - und viele früher deutsche oder österreichische Juden waren dies - konnten weiter recht problemlos Spanien legal passieren. Eine Verschlechterung bedeuteten die Regelungen für französische Juden, da ihr Land unter die kriegführenden Staaten eingereiht wurde.

Gerade wenn man unterstellt, dass die Verordnung mit auf deutschen Einfluss zurückzuführen ist, dürfte ihr Ziel nicht die Erschwerung des Transits jüdischer Flüchtlinge gewesen sein. ${ }^{22}$ Wir haben weiter oben gesehen, dass es zu diesem Zeitpunkt weiterhin deutsche Politik war, die Emigration von Juden, besonders aus dem Reichsgebiet, trotz der kriegsbedingten Probleme zu fördern. Sinn der Verordnung dürfte vielmehr gewesen sein, den legalen Transit von wehrfähigen Männern aus den von Deutschland besetzten Staaten - hier war besonders an Frankreich, die Niederlande und Belgien zu

S. 489, zufolge galt die Visite hauptsächlich den Sicherheitsvorkehrungen beim bevorstehenden Treffen Francos mit Hitler, doch habe man auch die längerfristige $\mathrm{Zu}$ sammenarbeit der Gestapo mit der spanischen Polizei besprochen. Preston stützt sich für Letzteres auf den Bericht des UNO-Sicherheitsrates über Spanien aus dem Jahr 1946, der zwar nicht gänzlich unzuverlässig, aber auch nicht frei von propagandistischen Behauptungen ist. Weitere Quelle Prestons ist Sekundärliteratur. Es muss daher offen bleiben, ob und ggf. wie Himmler auf Spaniens Flüchtlingspolitik Einfluss nahm.

${ }^{21}$ Der Erlass mit dem Zusatz der Botschaft in Frankreich in: AGA, AAEE, 11768. Marquina/Ospina, S. 148f. kannten diesen Zusatz nicht, so dass in ihrer Darstellung der Erlass eine ganz andere Bedeutung bekommt.

${ }^{22}$ Mit dieser Interpretation widersprechen wir der von Marquina/Ospina, S. 149: "Las medidas, en la práctica, significaban desanimar cualquier intento de éxodo.» 
denken - zu unterbinden. Dies lag im deutschen Interesse, und Spanien konnte sich damit als guter Verbündeter zeigen.

Ein Problem gibt es mit der Datierung dieser Ausnahmebestimmung. Im Exemplar des Konsulats Hendaye hat es den Anschein, als wenn sie in direktem Zusammenhang mit dem Erlass vom 8. Oktober 1940 formuliert worden sei. In einem Schreiben des Madrider Außenministeriums von Mitte Dezember d. J. wurde der Zusatz hingegen auf den 14. November datiert. ${ }^{23} \mathrm{Ab}$ diesem Datum galt dem Brief zufolge eine weitere Modifikation des Ursprungserlasses: Franzosen unter 18 oder über 40 Jahren wurden den Bürgern nicht kriegführender Staaten hinsichtlich der Transitvisa gleichgestellt, konnten sie also direkt vom spanischen Konsul erhalten. Dies erleichterte natürlich auch französischen Juden den Transit.

Weitere Veränderungen folgten am 28. und 30. Dezember $1940 .{ }^{24}$ Überliefert ist nur der Letztere der beiden Erlasse. Die Verordnung vom 28. Dezember ist, da als «Vertraulich» gekennzeichnet, nicht in der gedruckten Erlasssammlung des Spanischen Außenministeriums enthalten und auch sonst nicht auffindbar. ${ }^{25}$ Der zwei Tage später folgende Erlass sollte diesen nur ergänzen. Wir wissen also nicht, welche womöglich restriktiven Regeln ab dem 28. Dezember galten. Der uns zugängliche, zwei Tage jüngere Erlass brachte für die Transitvisa zwei Neuerungen. Der Besitz eines portugiesischen Transit- oder Einreisevisums war weiterhin unabdingbare Voraussetzung, doch sollte nun darauf gedrängt werden, dass die Antragsteller auch die Fahrkarten für ihre Schiffsreise von Portugal zum Zielland vorlegten. Portugal habe in letzter Zeit mehrfach Flüchtlinge an der Grenze abgewiesen, die zwar das portugiesische Visum, nicht aber die Fahrkarte besaßen. Eine zwingende Voraussetzung für die Erteilung eines spanischen Transitvisums war dies aber nicht. Auf das Problem war schon zuvor von der spanischen Grenzpolizei hingewiesen worden. Ihr zufolge betraf es nur jüdische Flüchtlinge. Von ihnen verlangte Portugal den Besitz der Schiffsbillets; zudem musste das Schiff schon in einem portugiesischen Hafen vor Anker liegen. Waren die Bedingungen nicht erfüllt, wurden die Juden von Portugal abgewiesen und

23 AMAE, R 1102/35: Spanisches Außenministerium an Militärkommandant Irun, 19. Dez. 1940.

${ }^{24}$ AMAE, Handbibliothek der Archivverwaltung, Erlasssammlung (ohne Signatur): Runderlass vom 30. Dez. 1940. Avni, Spain, waren die hier geschilderten Einzelheiten unbekannt, so dass er S. 74 - und in der Folge auch Zur Mühlen, S. 87 - davon ausgeht, dass seit dem 11. November 1940 generell Transitvisa nur nach Rückfrage in Madrid ausgestellt werden durften.

${ }^{25}$ Die Zitate von Marquina/Ospina, S. 164f., als deren Quelle sie die Erlasse vom 28. und 30. Dezember 1940 angeben, stammen ausnahmslos nur aus dem letzteren Erlass. Entgegen dem Anschein, den sie erwecken, konnten auch sie den Erlass vom 28. Dezember nicht auffinden. 
mussten auch Spanien in Richtung Frankreich verlassen. ${ }^{26}$ Dieses Problem sollte mit der Neuregelung beseitigt werden; Madrid unterschied aber, anders als Portugal, auch hier nicht zwischen Juden und Nichtjuden.

Die zweite Neuerung bedeutete hingegen i.w. eine Liberalisierung der Vergabe von Transitvisa. Direkt beim spanischen Konsul konnten nun alle Personen das Visum beantragen, die nicht Bürger kriegführender Staaten zwischen 18 und 30 Jahren, also im wehrfähigen Alter, waren. Damit war gegenüber dem Stand vom 14. November 1940 nicht nur für die Franzosen die Altersgrenze um zehn Jahre gesenkt worden. ${ }^{27}$ Die Bürger der anderen kriegführenden Staaten konnten nun ebenfalls auf ein einfach zu besorgendes Transitvisum hoffen, sofern sie nicht in diesem Alter waren. Andererseits wurden die Bestimmungen für Bürger kriegführender Staaten zwischen 18 und 30 verschärft. Ihnen hatte seit Oktober noch der - beschwerliche - Weg offen gestanden, beim Außenministerium in Madrid ein Transitvisum zu beantragen. Nun wurde bestimmt, dass diese Personen erst gar keine Anträge stellen dürften. Dies bestätigt, dass vorrangiges Ziel der Erlasse war, wehrfähigen Männern die Flucht aus Frankreich unmöglich zu machen; im übrigen aber ließ Spanien jeden zum Transit zu, der Garantien dafür besaß, nicht im Land zu bleiben.

Ende 1940 sah die rechtliche Situation also so aus, dass über Einreisevisa in Madrid entschieden wurde, Transitvisa aber von den örtlichen Konsuln vergeben wurden. ${ }^{28}$ Von beiderlei Arten von Visa ausgeschlossen waren Bürger kriegführender Staaten zwischen 18 und 30 Jahren. $^{29}$ Prinzipiell abgelehnt wurden von spanischer Seite Gruppenvisa. Madrid wollte jeden Antragsteller gesondert überprüfen können. ${ }^{30}$

${ }^{26}$ AGA, AAEE, 11769: Aktennotiz Jefatura de la Frontera Norte de España, Irún, 18. Dez. 1940.

27 Dies war schon am 23. Dezember der Botschaft in Vichy mitgeteilt worden. Vgl. AMAE, R 1102/35.

28 Da Marquina/Ospina der Erlass vom 1. Mai 1940 unbekannt war, hatten sie den Eindruck, dass nun erstmals die Entscheidung über Einreisevisa nach Madrid verlagert worden sei. Dies interpretierten sie als restriktive Maßnahme. Ein anderes Problem ihrer Darstellung ist, dass sie den selbst schon recht konfus formulierten Erlass noch unverständlicher und widersprüchlicher wiedergeben. Vgl. S. 164f.

${ }^{29}$ Vergeblich sucht man in den Erlassen eine Differenzierung zwischen Männern im wehrdienstfähigen Alter und Frauen.

${ }^{30}$ Beispiele bei Marquina/Ospina, S. 146f.; vgl. auch Tusell 1995, S. 579. Dies dürfte auch die Ursache für die Probleme, die Gruppentransporte Luxemburger Juden an der spanischen Grenze hatten, gewesen sein. Ino Arndt zufolge wanderten zwischen August 1940 und Oktober 1941387 Luxemburger Juden nach Portugal und 50 nach Spanien aus. Vgl. Ino Arndt, Luxemburg. In: Benz, Wolfgang ( $\mathrm{Hg}$.): Dimension des Völkermords. Die Zahl der jüdischen Opfer des Nationalsozialismus. München 1991, S. 95104, S. 101. Bauer, Yehuda: American Jewry and the Holocaust. The American Jewish Joint Distribution Committee, 1939-1945. Detroit 1981, S. 54f., berichtet, dass am 
Mitte Februar 1941 kam es zu einer vorläufig letzten Veränderung der Rechtslage. Wahrscheinlich hatte es Unklarheiten darüber gegeben, ob die Fahrt zu einem Schiff, das in einem spanischen Hafen lag, Transit oder Einreise bedeutete. Außenminister Serrano Suñer bestimmte am 18. Februar, dass dafür ein gesondertes Visum eingeführt wurde, zehn bis dreißig Tage gültig. Es konnte direkt von den spanischen Konsulaten ausgestellt werden. Welche sonstigen Anforderungen an die Visumerteilung geknüpft waren, lässt sich leider nicht sagen, denn im Erlass heißt es nur, dass die Anforderungen, die «für Pässe gelten», auch hier anzuwenden seien, ohne dass wir wissen, was darunter zu verstehen ist. Ziel des Erlasses war auch eine stärkere Nutzung der von spanischen Häfen abgehenden Schiffahrtslinien. Daher wurden sie im Erlass namentlich genannt. Neben zwei nationalen Gesellschaften war dies ein US-amerikanisches Unternehmen. ${ }^{31}$

Bereits wenige Monate später, am 23. Oktober 1941, folgte eine Anordnung der Generaldirektion für Seeverkehr im Industrie- und Handelsministerium, mit der spanischen Schiffahrtsgesellschaften die Beförderung von Juden nach Nordamerika und Kuba untersagt wurde. Grund dafür war, dass einige Juden nach der Ankunft in den USA vor Gericht Entschädigungen für Wucherpreise, die sie für die Schiffspassage hatten bezahlen müssen, einforderten. Die Richter hatten dies offenkundig akzeptiert und als Sicherheit betroffene Schiffe beschlagnahmt. ${ }^{32}$ Wir wissen nicht, wie lange dieses Verbot in Kraft blieb. Jedenfalls stand jüdischen Flüchtlingen weiterhin die Möglichkeit offen, von Lissabon abzureisen.

Umstritten ist, ob die spanischen Behörden die Religion oder «Rasse» der Antragsteller interessierte. In den zahlreichen Anträgen - meist betrafen sie den Transit -, die das Konsulat Hendaye 1940, als es nicht zur direkten Ausstellung der Papiere berechtigt war, an das Außenministerium in Madrid weiterleitete, gibt es keinerlei entsprechende Angaben. ${ }^{33}$ Avni hingegen be-

11. November 1940203 Luxemburger Juden mit zweifelhaften Visa von Spanien an der Grenze abgewiesen wurde, jedoch später - unklar ist, ob in kleineren Gruppen oder individuell - nach Portugal reisen konnten. Die wahrscheinlich letzte Gruppe traf Anfang November 1941 an der spanischen Grenze ein. Der spanische Grenzkommandant teilte dem Konsulat im französischen Grenzort Hendaye mit, dass auf Anweisung der Sicherheitspolizei DGS den 122 Juden aus Luxemburg (viele von ihnen hatten die deutsche Staatsangehörigkeit) Transitvisa auszustellen seien. Vgl. AGA, AAEE, 11769: Comandancia Militar del Bidasoa e Inspección de los Servicios de Frontera del Norte de España an Spanischen Konsul Hendaye, 5. Nov. 1941. Die Anweisung zeigt einerseits Spaniens Bereitschaft zur Gewährung von Transit, zugleich aber auch, dass Gruppenvisa nur ausnahmsweise erteilt wurden.

31 AGA, AAEE, 1633: Spanisches Außenministerium an Spanische Botschaft Vichy, 18. Febr. 1941.

32 AMAE, R 1190/83; American Jewish Year Book 44 (1942/43), S. 231; Marquina/ Ospina, S. 166.

33 AGA, AAEE, 11770. 
hauptet das Gegenteil, stützt sich dabei aber auf einen einzigen Augenzeugen. Juden seien jedoch, so schreibt auch er, nicht wegen ihrer Religion vom Transit ausgeschlossen worden, die Frage habe also keine praktischen Auswirkungen gehabt. ${ }^{34}$ Marquina/Ospina berichten, dass Visaanträge von Juden den Zusatz «Israelitas»» getragen hätten. ${ }^{35}$ Nach unseren Erhebungen in den Archiven waren es unter den Anträgen auf Transitvisa höchstens Ausnahmefälle, in denen die Religion erfragt wurde. Deswegen ist es aber schwer, die Zahl jüdischer Flüchtlinge, die Spanien im Transit passierten, genau zu bestimmen. Auch bei den Einreiseanträgen haben wir keine gesehen, in denen die Religion erfragt wurde; die Bestimmungen der einschlägigen Erlasse machen aber deutlich, dass in diesem Bereich die Religion von Bedeutung war, weil Spanien Juden von der Einreise ausschließen wollte.

Ein weiteres Problem muss hier angesprochen werden. Zu den von Spanien geforderten Garantien für den Transit gehörte auch der Nachweis ausreichender finanzieller Mittel. Nun war Spanien damals kein teures Land. Trotzdem rechnete man mit Transitkosten von 60-80 US-\$. Wer diese Summe nicht vorweisen konnte, lief Gefahr, wegen Landstreicherei aufgegriffen zu werden. ${ }^{36}$ Aber Mitte 1942 durften an der spanischen Grenze, so berichtet Avni unter Berufung auf jüdische Hilfsorganisationen, US-Dollar nicht mehr gegen Peseta getauscht werden. Mochten die Flüchtlinge auch genügend Devisen haben, es fehlte ihnen an der nationalen Münze. Der Transit war unmöglich, sie mussten nach Frankreich zurückkehren. Avni erwähnt leider nicht, wie sich diese Angelegenheit weiter entwickelte. ${ }^{37}$ Jedenfalls gab es auch danach Transitreisende.

\section{Zurückweisung illegal eingereister Flüchtlinge ab Juni 1940}

Bisher haben wir uns mit legalen Grenzgängern beschäftigt; nun wollen wir den Gesichtskreis erweitern und auch das Schicksal illegaler Flüchtlinge einbeziehen. Vorweg muss betont werden, dass es auch hier keine offizielle Unterscheidung zwischen Juden und Nichtjuden gab und daher die folgenden Ausführungen für alle Flüchtlinge gelten.

Über Erlasse der spanischen Regierung für den Umgang mit illegalen Grenzgängern gibt es keine Informationen ${ }^{38}$ In der Praxis ergab sich folgen-

${ }^{34}$ Avni, Spain, S. 75.

${ }^{35}$ Marquina/Ospina, S. 149.

${ }^{36}$ Zur Mühlen, S. 90.

${ }^{37}$ Avni, Spain, S. 75.

${ }^{38}$ Sowohl das Archiv des Innenministeriums als auch das der Sicherheitspolizei DGS erklärten, sie besäßen keine Unterlagen darüber. Auch in den Archiven der 
des Bild: Illegal eingereist war nach spanischer Auffassung nicht nur, wer keine oder gefälschte Papiere besaß, sondern auch wer nicht zügig das Land im Transit passierte oder wer die Devisenvorschriften nicht beachtet hatte. Letzteres konnte darin bestehen, das mitgeführte Bargeld unzutreffend deklariert zu haben oder über zu wenig Geld für die Transitkosten zu verfügen. ${ }^{39}$ In der Regel war das Problem der illegalen Flüchtlinge, dass sie kein portugiesisches Transitvisum besaßen, und Letzteres fehlte meist deswegen, weil sich kein Land zur definitiven Aufnahme des Flüchtlings bereit erklärt hatte. Das größte Verhaftungsrisiko bestand im engeren Grenzbereich und in den Zügen zu den nächstgelegenen großen Städte. Aber auch in einer Stadt wie Barcelona waren die illegal eingereisten Flüchtlinge nicht sicher; Emilienne Eychenne berichtet, dass sich die spanische Polizei häufig in der Nähe des britischen Konsulats postierte und Flüchtlinge, die dort um Papiere nachsuchten, nach dem Verlassen des Gebäudes festnahm. ${ }^{40}$ Die Verhaftung durch die spanische Polizei bedeutete nicht automatisch die Auslieferung an die Deutschen oder an die Franzosen. Die Kriterien, nach denen entschieden wurde, ob illegale Flüchtlinge, die auf spanischer Seite verhaftet worden waren, ausgeliefert oder im Land festgehalten wurden, sind heute nicht mehr zu rekonstruieren.

Im Sommer 1942 wuchs die Zahl der illegalen Flüchtlinge kräftig. Auslöser war der Beginn der Deportationen von Juden aus Frankreich Mitte Juli 1942. Die meisten Flüchtlinge waren staatenlos, denn diese Juden wurden zuerst von den Deportationen erfasst. Durch die große Zahl stieg die Gefahr, dass die illegalen Flüchtlinge nach der Festnahme ausgeliefert werden würden. Daher intervenierte die US-Botschaft in Madrid und erhielt im August 1942 vom spanischen Außenministerium die Zusicherung, dass kein illegaler Flüchtling gegen seinen Willen zurückgesandt werde. Die Wirkung des Versprechens wurde aber dadurch geschmälert, dass sich das Außenministerium nicht immer gegen die Sicherheitspolizei und das Innenministerium durchsetzen konnte. Immerhin kam es in den ersten Monaten nach der Übereinkunft nur in vereinzelten Fällen zu Zurückweisungen, initiiert von lokalen Behörden. Ende Oktober 1942 gab es aber in Barcelona Indizien, dass die Polizei eine generelle Ausweisung von illegalen Flïchtlingen vorbereitete. An der Grenze erfolgten nun vermehrt Fälle von Zurückweisungen. Erneut intervenierte die USA-Botschaft beim spanischen Außenministerium. ${ }^{41}$

«Presidencia del Gobierno» (Staatskanzlei) und des Außenministeriums konnten wir keine einschlägigen Dokumente finden.

${ }^{39}$ Zur Mühlen, S. 95.

${ }^{40}$ Eychenne, Emilienne: Les Portes de la Liberté. Le franchissement clandestin de la frontière espagnole dans les Pyrénées-Orientales de 1939 à 1945. Paris 1985, S. 133.

41 Avni, Spain, S. 94, 98-102; Bauer, American Jewry, S. 208. 
Bevor es zu einer spanischen Antwort kam, wurde die Situation durch die alliierte Landung in Nordafrika am 8 . November und die folgende Besetzung Südfrankreichs durch deutsche Truppen völlig verändert. Zahlreiche Franzosen, besonders im wehrpflichtigen Alter, überschritten nun die Grenze, um einer Zwangsarbeit in Deutschland zu entgehen und/oder sich den alliierten Streitkräften anzuschließen. Für die Alliierten stieg die Bedeutung der Flüchtlingsfrage. Zudem bat auch der Botschafter Vichy-Frankreichs in Madrid, Piétri, die spanische Regierung, keine illegalen Flüchtlinge mehr zurückzuschicken. Am 17. November 1942 wurde dieser Bitte entsprochen. ${ }^{42}$

Auf der Nordseite der Pyrenäen verstärkten Deutsche und Franzosen nun die Grenzkontrollen. Am 18. Februar und am 3. April 1943 wurden alle Juden aus den Grenzdepartements ausgewiesen. In einer Sperrzone entlang der Grenze durften sich nur noch Besitzer einer gesonderten Erlaubnis aufhalten. ${ }^{43}$ Zugleich übte Deutschland Druck auf Spanien aus. Zusammen mit der weiterhin hohen Zahl illegal einreisender Flüchtlinge führte dies die spanische Regierung - konkret: Franco, der in diesem Fall den Ministerrat nicht konsultierte - dazu, am 25. März 1943 die Schließung der Pyrenäengrenze für Flüchtlinge ohne Transitvisa anzuordnen. ${ }^{44}$ Die Grenzkontrollen sollten verstärkt, illegale Flüchtlinge verhaftet werden. Spanien weigerte sich, eine Zusicherung zu geben, dass sie nicht abgeschoben würden. ${ }^{45}$ Die Alliierten reagierten scharf, denn für sie ging es darum, dass alliierten Soldaten weiterhin der Fluchtweg über Spanien offen stand. Außenminister Jordana konnte daraufhin regierungsintern erreichen, dass Soldaten nicht zurückgewiesen werden würden und das Flüchtlingsproblem insgesamt neu überdacht werde, was er den Alliierten mitteilte. Trotz dieser Zusage kam es zur Zurückweisung von Flüchtlingen. Am 7. April warnte Churchill den spanischen Botschafter, dass dies die beiderseitigen Beziehungen belaste. Ähnlich äußerte sich das Außenministerium in Washington. Auch der päpstliche Nuntius und der argentinische Botschafter in Madrid appellierten auf Bitten des US-Botschafters an Jordana. Dieser Druck führte nun wirklich zur Aufhebung der Grenzsperre. Erneut wurde allen, auch den illegalen Flüchtlingen, zugestanden, im Land zu bleiben, bis sie eine Möglichkeit zur Weiterreise gefunden

${ }^{42}$ Avni, Spain, S. 77, 102f.; Bauer, American Jewry, S. 209.

43 Avni, Spain, S. 103.

${ }_{44}$ Tusell, 1995, S. 380.

${ }^{45}$ Cruz Roja Española, Archivo, Madrid: Caja 938, exp. 15: Ministerio de Asuntos Exteriores, Dirección de Política Exterior, Europa, Doussinague, an Presidente de la Asamblea Suprema de la Cruz Roja Española, Madrid, 25. März 1943; AGA, AAEE, 7568: Spanisches Außenministerium an Spanische Botschaft London, 30. März 1943; Foreign Relations of the United States. Diplomatic Papers, 1939-1945. 1943, Bd.I. Washington D.C. 1963, S. 276f.: Ambassador in Spain (Hayes) an Secretary of State, 29. März 1943. 
hatten. Vereinzelte Zurückweisungen an der Grenze, die es auch in der Folgezeit noch gab, waren auf die Initiative lokaler Behörden zurückzuführen, entsprachen aber nicht der Regierungslinie. ${ }^{46}$

Vollständige Angaben, wie viele illegale Flüchtlinge in Spanien interniert und wie viele abgeschoben wurden, liegen nicht vor. ${ }^{47}$ Zahlen gibt es für das Departement Pyrénées-Orientales, das sich von Andorra bis zum Mittelmeer erstreckt. Bis November 1942 war es nicht von deutschen Truppen besetzt. Dies und die Tatsache, dass gerade in Küstennähe die Berge relativ leicht zu passieren waren, führte dazu, dass das Departement bis zum Einmarsch der Deutschen von den illegalen Flüchtlingen bevorzugt wurde. In dieser Zeit gab es nach französischen Unterlagen und Augenzeugenberichten 352 Auslieferungen oder Zurückweisungen durch Spanien. Am zahlreichsten waren sie im April 1941. ${ }^{48}$ Folgen wir Eychennes Schätzung, dass bis November 1942 70\% der illegalen Flüchtlinge, die von Frankreich nach Spanien wechselten, dieses Departement passierten, käme man für die gesamte Grenze und diesen Zeitraum auf etwa 500 von Spanien ausgelieferte oder zurückgewiesene Flüchtlinge ${ }^{49} \mathrm{Im}$ folgenden Zeitraum bis zur Befreiung der Grenzdepartements im August 1944 liegt die Zahl der Auslieferungen und Zurückweisungen bei nur noch 28. Da Eychenne den Anteil der Pyrénées-Orientales an den illegalen Grenzübertritten für diese Phase nur noch auf $30 \%$ veranschlagt, kann man die Gesamtzahl der Auslieferungen auf etwa 100 schätzen. Ganz eindeutig geht dieser Rückgang auf alliierten Druck zurück. ${ }^{50}$

\section{Das Schicksal von Flüchtlingen, die in Spanien verhaftet wurden}

Verhaftete Flüchtlinge, die nicht ausgeliefert wurden, kamen in das regionale spanische Gefängnis. Die Zustände dort waren in vielen Fällen sehr schlecht. Dies lag weniger an Schikanen als an der generellen Versorgungskrise nach

46 Avni, Spain, S. 104f.

47 So auch: Avni, Haim: España y Portugal. Su actitud respecto de los refugiados judíos durante la era nazi. In: Klich, Ignacio/Rapoport, Mario (Hg.): Discriminación y Racismo en América Latina. Buenos Aires 1997, S. 255-271, hier: S. 261.

48 Eychenne, Portes, S. 119, 162. Im Falle von Auslieferungen wurden die Betroffenen an die Behörden des Nachbarstaates überstellt (bis zum 11. November 1942 waren dies die Franzosen, ab diesem Tag deutsche Stellen), bei Zurückweisungen wurden Flüchtlinge unmittelbar an der Grenze zurückgeschickt, ohne dass dies der Gegenseite mitgeteilt wird.

${ }^{49}$ Eychenne, Portes, S. 189. Die Schätzung unterstellt natürlich, dass illegale Grenzübertritte und Auslieferungen auch in den anderen Grenzregionen im selben Verhältnis zueinander standen. Dies vorauszusetzen ist problematisch, hilft aber bei der Ermittlung der ungefähren Größenordnung von Auslieferungen bzw. Zurückweisungen.

so Eychenne, Portes, S. 119, 146, 189. 
Ende des Bürgerkriegs. Bis Ende 1942 nahmen dies immer wieder Flüchtlinge zum Anlass, freiwillig in das unbesetzte Frankreich zurückzukehren. Diese Bereitschaft endete mit der Besetzung des ganzen Landes im November 1942. ${ }^{51}$ Aus den Gefängnissen kamen die Männer nach etwa drei bis neun Wochen in das Lager Miranda de Ebro in der Nähe von Burgos (Nordspanien). Das Lager war gegen Ende des Bürgerkriegs für politische Gegner Francos eingerichtet worden. Seit 1940 befanden sich dort Flüchtlinge, die illegal ins Land gekommen waren oder die trotz gültiger Papiere nicht nach Portugal hatten weiterreisen können. ${ }^{52}$ Die Dauer des Aufenthalts in Miranda de Ebro konnte sehr variieren, von wenigen Tagen bis zu mehreren Monaten. ${ }^{53}$ Im Lager lebten die Häftlinge in fensterlosen Unterkünften, ihre Kleidung schützte nicht vor der Kälte, die in dieser Gegend Spaniens im Winter üblich ist. Es gab aber keine wahllosen Misshandlungen und Juden wurden in der Regel nicht anders behandelt als die übrigen Häftlinge. ${ }^{54}$ US-Bürger und Staatsangehörige des Commonwealth, besonders wenn sie wehrfähige Männer waren, hatten die größte Chance, bald freigelassen zu werden und auszureisen. Daher gaben sich nicht wenige Flüchtlinge als «Frankokanadier» aus; von spanischer Seite wurde dies nicht näher überprüft. Sie wurden zwar in Spanien meist von alliierter Seite unterstützt, aber bei den Repatriierungsaktionen wurde von den Konsulaten aus Furcht vor deutschen Spionen stärker kontrolliert, so dass diese Flüchtlinge im Land bleiben mussten. Auch das Alter wurde immer wieder falsch angegeben, da die Flüchtlinge befürchteten, wehrfähige Männer würden länger festgehalten. Von Oktober bis Dezember 1942 stieg die Zahl der Insassen des Lagers Miranda de Ebro von

51 Ebd., S. 144f.

52 Über das Lager Miranda gibt es bisher noch keine grundlegende Studie. Die Informationen über die Quellenlage sind widersprüchlich. Einerseits schrieb die Madrider Tageszeitung El País am 26. Juni 1994, dass trotz aller Bemühungen der Gemeindeverwaltung von Miranda de Ebro die Akten des Lagers nicht aufzufinden waren, auch nicht in Militärarchiven. Andererseits verzeichnet der Guía de Archivos Militares Españoles. Madrid 1995, S. 75, für das Archivo General Militar de Guadalajara 40 Aktenbündel («legajos») über das Lager für die Jahre 1936-1945. Da die Geschichte des Lagers außerhalb des Themenbereiches dieser Untersuchung liegt, haben wir die Akten nicht konsultiert.

53 Zur Mühlen, S. 96f.; vgl. auch: Avni, Spain, S. 76f.; Bauer, American Jewry, S. 208.

54 Im AGA, AAEE, 7568, befindet sich ein Fotoalbum mit 42 Aufnahmen aus dem Lager Miranda. Sie waren von staatlicher Seite gemacht worden. Das Album wurde im Dezember 1943 der Spanischen Botschaft in London übersandt, als Reaktion auf kritische Äußerungen des dortigen niederländischen Botschafters über das Lager. Die Fotografien zeigen ein sauberes Lager, Einrichtungen wie das Hospital oder die Friseurstube und Außenaufnahmen der Baracken, jedoch keine Innenaufnahmen. In Miranda waren zahlreiche Niederländer interniert, die über Belgien und Frankreich gekommen waren. Ihr Ziel war Großbritannien, wo sie sich den alliierten Truppen anschließen wollten. 
1.400 auf 3.500, womit es überfüllt war. Ab Ende 1942 galt für Offiziere im aktiven Dienst, also alliierte Soldaten, dass sie in einer Militärbasis konzentriert werden sollten. Die übrigen illegalen Flüchtlinge würden freigelassen so die damals mit den Alliierten vereinbarte Neuregelung -, sofern sie für sich selbst sorgen könnten oder jemand dafür aufkomme. Während von dieser Maßnahme nicht erfasste Männer im wehrfähigen Alter in Miranda blieben, wurden Frauen in den Gefängnissen von Figueras (nördlich von Barcelona), Barcelona und Madrid konzentriert. ${ }^{55}$

Anfang 1943 wurden nach einem Hungerstreik, mit dem gegen die schlechte Unterbringung und Verpflegung in Miranda de Ebro protestiert wurde, viele Flüchtlinge entlassen. Diejenigen aus alliierten Ländern wurden evakuiert, Staatenlose und Flüchtlinge aus deutsch besetzten Staaten von Hilfsorganisationen unterstützt und ihnen Zwangsaufenthaltsorte zugewiesen. Im Mai 1943 zählte das Lager 1.100-1.200 Insassen. Bald aber füllte es sich wieder mit neuen Flüchtlingen. Bis Ende 1943 waren im Lager konstant um die 3.000 Häftlinge, jedoch sank die durchschnittliche Aufenthaltsdauer. Erneut konnten viele Flüchtlinge das Lager verlassen, nachdem ihre Konsulate die Repatriierung zugesagt hatten. ${ }^{56}$

Im September 1943 stimmte die spanische Regierung trotz deutscher Pressionen der Evakuierung von 16.000 französischen Flüchtlingen nach Nordafrika zu. ${ }^{57}$ Der Botschafter des Vichy-Regimes, Piétri, trat dieser Aktion nicht entgegen. ${ }^{58}$ Auch kam es erneut zur Freilassung staatenloser Häftlinge, sofern sie anschließend von Hilfsorganisationen betreut wurden. Bis zur Ausreise aus Spanien durften sie unter Polizeiaufsicht in Madrid und Barcelona leben..$^{59}$

Damit kommen wir zur Aktivität von jüdischen Hilfsorganisationen in Spanien. Um illegale Flüchtlinge, die inhaftiert waren, kümmerten sich 1940/ 41 die Frau des US-Botschafters Weddell und die des Militärattachés. Sie ließen aus Mitteln der US-amerikanischen jüdischen Wohlfahrtsorganisation JOINT, die offiziell in Spanien nicht tätig werden durfte, Häftlingen im Lager Miranda de Ebro etwas Unterstützung zukommen. Weddells Nachfolger Hayes untersagte bei seiner Amtsübernahme im April 1942 diese Tätigkeit. $^{60}$ Die Durchreise jüdischer Flüchtlinge wurde von den HICEM- und JOINT-

55 Avni, Spain, S. 77, 102f., 106, und Zur Mühlen, S. 98-101. Beide Autoren stïtzen sich auf Interviews mit Zeitzeugen. Vgl. auch Bauer, American Jewry, S. 209.

56 Avni, Spain, S. 77, 79, 106-108, 112 f.

57 Marquina/Ospina, S. 179.

58 Tusell, 1995, S. 536.

59 Avni, Spain, S. 122f.; Leshem, Perez (früher: Lichtenstein, Fritz): Rescue Efforts in the Iberian Peninsula. In: Leo Baeck Institute Yearbook XIV (1969), S. 231-256, S. 246, nennt als Zwangsaufenthaltsorte Kleinstädte in Nordspanien.

${ }^{60}$ Avni, Spain, S. 78. 
Büros in Marseille (bis Ende 1942) und Lissabon unterstützt. In Spanien selbst konnten sie sich anfänglich an einige wenige Vertrauenspersonen wenden, die ohne Genehmigung tätig waren. Bis zum Verbot der Auswanderung von Juden im Oktober 1941 fungierte Fred Max Oberländer in Barcelona als Berater für diejenigen, deren Emigration von der Reichsvereinigung der Juden in Deutschland organisiert worden war. Bezahlt wurde Oberländer vom JOINT ${ }^{61}$ In einem großen Madrider Hotel half ein Portier, der selbst jüdischer Flüchtling war, in Irun der Agent eines deutschen Reisebüros. Etwas offizieller wurde die Arbeit ab 1941, als der Portugiese Samuel Sequerra aktives Mitglieder der Jüdischen Gemeinde Lissabon - nach Barcelona kam. Nach außen war er dortiger Vertreter des portugiesischen Roten Kreuzes, de facto Repräsentant des JOINT in Barcelona. Die spanischen Behörden duldeten dies, doch wurde Sequerra von der Polizei überwacht und sein Büro im August 1942 durchsucht. ${ }^{62}$ Das portugiesische Konsulat in der katalanischen Hauptstadt unterstützte ihn. Da er sich auch um nichtjüdische staatenlose Flüchtlinge kümmerte, waren bald die Konsulate alliierter Staaten zur Kooperation bereit. Die deutliche Zunahme der Zahl illegaler Flüchtlinge ab der zweiten Jahreshälfte 1942 stellte Sequerra aber vor Aufgaben, denen sein Büro nicht mehr gewachsen war. Auch die alliierten Konsulate waren überfordert, hatten sie es doch mit vielen Flüchtlingen zu tun, die eine falsche Staatsangehörigkeit angaben. In der US-Botschaft wurde eine Flüchtlingsabteilung unter Miles Bond eingerichtet. Geld und Lebensmittel erhielt sie vom Roten Kreuz der USA. Auf längere Sicht noch bedeutender waren die Gespräche, die der US-Botschafter Hayes Ende 1942 mit Außenminister Jordana führte. Ihr Ziel war die Zulassung eines gemeinsamen Büros USamerikanischer Hilfsorganisationen. Da sich die spanische Regierung von einer derartigen Einrichtung die beschleunigte Ausreise der Flüchtlinge erhoffte, erteilte sie Anfang 1943 die Genehmigung. Leiter der Vertretung wurde David Blickenstaff von der Quäker-Organisation American Friends Service Committee. Auch der JOINT, der anfangs 2/3 der Kosten trug, war mit seiner Ernennung einverstanden. 1944 schlossen sich weitere US-amerikanische Hilfsorganisationen der Einrichtung an. Blickenstaff, Jahrgang 1915, kannte das Land von seiner Tätigkeit während des Bürgerkrieges. Sein erstes Büro befand sich in einem Raum der USA-Botschaft; ab 10. April 1943 hatte die Representation in Spain of American Relief Organizations eigene Räume in einem Gebäude zwischen der Botschaft und dem Sitz des spanischen Roten

${ }^{61}$ Ebd., S. 261.

62 Ebd., S. 77f.; Avni, España, S. 262, 264; Bauer, American Jewry, S. 49; Willson, John P.: Carlton J. H. Hayes, Spain, and the Refugee Crisis, 1942-1945. In: American Jewish Historical Quarterly 62 (Dezember 1972), S. 99-110, S. 103. 
Kreuzes. ${ }^{63}$ Hauptsächliche Zielgruppe von Blickenstaff waren Staatenlose dies waren meist Juden - und Flüchtlinge mit Pässen deutsch besetzter Länder; er kümmerte sich aber auch um repatriierte spanische Juden. ${ }^{64}$

1943/44 half Sequerra bei der Flucht von einigen hundert jüdischen Kindern aus Frankreich, die von der Union Générale des Israélites de France organisiert wurde.

We were constantly in contact with the border and when a group of Jews, children or adults had crossed the French-Spanish border illegally, we were notified of it by telephone by the Spanish frontier police. We then went to the border and posted a guarantee for the refugees. After the Jewish refugees were kept there during a few days to check their papers, they were freed and we took them to Barcelona. [...] We sent the children to Palestine, to the United States and to Canada. ${ }^{65}$

Damit ist ein letzter Gesichtspunkt angesprochen, der die spanische Flüchtlingspolitik beeinflusste: die Aufnahme von Flüchtlingen, die kein Einreisevisum eines überseeischen Landes erhalten hatten, in alliierten Lagern. Dieses Problem stellte sich besonders ab Ende 1942, als die legale Ausreise aus Vichy-Frankreich unmöglich geworden war. Somit konnte sich auch kein Flüchtling mehr bereits in Frankreich das Visum eines Aufnahmelandes besorgen. Flüchtlinge aus alliierten Ländern wurden relativ schnell aus Spanien repatriiert. Bürger deutsch besetzter Staaten, deren Exilregierungen mit den Alliierten verbündet waren, konnten, jedenfalls wenn sie Männer und im wehrfähigen Alter waren, ebenfalls bald ausreisen, entweder nach Nordafrika oder nach Großbritannien, wo sie sich den alliierten Truppen anschlossen. Zum Problem für Spanien wurden die staatenlosen Flüchtlinge, meist Juden, die nur schwer ein Aufnahmeland fanden. Auf der Bermuda-Konferenz im April 1943, die zur Lösung des Flüchtlingsproblems einberufen worden war, waren sich die USA und Großbritannien einig, dass dadurch die Aufnahmefähigkeit und -bereitschaft Spaniens für weitere Flüchtlinge blockiert werde, weswegen schnelle Abhilfe erforderlich sei. Daher wurde beschlossen, Flüchtlinge von der Iberischen Halbinsel in neu zu errichtende Lager in Nordafrika und Palästina zu evakuieren. Es war die Rede von 14.000 Franzosen und 6.000-8.000 Juden. Die Realisierung dieses Beschlusses ließ aber fast ein Jahr auf sich warten, da sowohl US-Militärs als auch französische Stellen Bedenken hatten, in arabischen Gebieten Lager für jüdische Flüchtlinge zu eröffnen. ${ }^{66}$ Schließlich wurde es von der UNRRA, der UN-Flüchtlingshilfsorganisation, in Fedala nahe Casablanca eingerichtet.

${ }^{63}$ Avni, Spain, S. 99, 114f.; Beaulac, Willard Leon: Franco. Silent ally in World War II. Carbondale/USA 1986, S. 174f.; Hayes, S. 122f.; Lion Lewin, Madrid, S. 35.

${ }_{64}$ Vgl. Bauer, American Jewry, S. 211, 478 Anm. 27.

65 Amipaz-Silber, S. 226. Auch die Armée Juive verfügte 1944 über eine Organisation, die Juden über Spanien nach Palästina brachte; vgl. ebd., S. 364.

${ }^{66}$ Avni, Spain, S. 118-121; Willson, S. 104f. 
Die Evakuierung der staatenlosen Flüchtlinge - nicht nur nach Fedala wurde von Blickenstaffs Büro organisiert. Über die Gesamtzahl derer, die davon erfasst wurden, liegen widersprüchliche Angaben vor. Willson zufolge verließen 19433.500 staatenlose Flüchtlinge - in der Regel waren dies Juden - Spanien. ${ }^{67}$ Nachrichten über Ausreisen von ganzen Gruppen haben wir aber erst für den Januar 1944 (s.u.). Womöglich bezieht sich Willsons Zahl auf die individuelle Weiterreise nach Portugal, dürfte dann aber zu hoch liegen. Wyman berichtet erst für 1944 von Ausreisen staatenloser Flüchtlinge. Im Laufe des Jahres seien etwa 1.800 nach Kanada, Palästina, in die USA und nach Fedala evakuiert worden; Anfang 1945 hätten sich noch etwa 1.000 Flüchtlinge in Spanien aufgehalten. ${ }^{68}$

Weniger Unklarheit herrscht über die Zahl der nach Fedala Evakuierten. Avni gibt an, dass von Blickenstaffs Büro insgesamt 1.340 Flüchtlinge auf ihre Eignung überprüft wurden; Kriterien waren der Gesundheitszustand und eine Sicherheitsüberprüfung. Die französischen Behörden erlaubten 977 von ihnen die Einreise, aber nur 630 erreichten das Lager. ${ }^{69}$ Ursache für diese Differenz war, dass viele Flüchtlinge nicht nach Nordafrika evakuiert werden wollten. In Spanien lebten sie mittlerweile häufig in relativer Freiheit in festgelegten Aufenthaltsorten, nicht mehr in Lagern oder Gefängnissen, und wurden vom JOINT finanziell unterstützt. ${ }^{70}$

Das wichtigste Aufnahmeland für jüdische Flüchtlinge aus Spanien war Palästina. Dies war im Frühjahr 1943 von Wilfried Israel von der Jewish Agency in London vorbereitet worden, doch wurde sein Flugzeug bei der Rückreise von der Iberischen Halbinsel von der deutschen Luftwaffe abgeschossen und alle Unterlagen gingen verloren. Im Herbst 1943 erhielt Fritz Lichtenstein (später: Perez Leshem) von der Jewish Agency den Auftrag, Israels Arbeit fortzusetzen. Dabei musste er sowohl auf britischer als auch auf spanischer Seite Widerstände überwinden. Spanischen Quellen zufolge erklärte der britische Botschafter in Madrid, dass während der Dauer des Krieges eine genaue Sicherheitsüberprüfung der Kandidaten für die Einreise nach

67 Willson, S. 106.

68 Wyman, S. 253f.

69 Avni, Spain, S. 121. Auch das American Jewish Year Book 46 (1944/45), S. 310, berichtet, dass bis Mitte 1944600 Flüchtlinge aus Spanien in Fedala eingetroffen seien. Diesem Bericht zufolge kamen die Widerstände gegen ihre Einreise von den französischen Behörden, die eine Gefährdung der nationalen Sicherheit befürchteten. Jeder Flüchtling habe 16 Formulare ausfüllen müssen, fünf davon mussten durch ein Foto ergänzt werden, elf durch Fingerabdrücke.

70 Avni, Haim: The war and the possibilities of rescue. In: Cohen, Asher/Cochavi, Yehoyakim/Gelber, Yoav (Hg.): The Schoah and the War. New York 1992, S. 384-388, S. 384. 
Palästina erforderlich sei. ${ }^{71}$ In Portugal und Spanien stellte Lichtenstein Listen von Kandidaten zusammen. Nach Überwindung zahlreicher bürokratischer Hindernisse gelang es ihm, im Januar 1944 einen Transport auf dem Schiff Nyassa von Lissabon über Cadiz nach Palästina zu organisieren. Aus Portugal kamen 166 jüdische Flüchtlinge, aus Spanien und Tanger 564. Letztere gliederten sich so auf: 384 aus Barcelona, 138 aus Madrid, 42 aus dem Lager Miranda. Etwa $40 \%$ der 564 waren Juden aus dem «Großdeutschen Reich», einige weitere hatten dort bis 1933 gelebt und waren 1940 aus Frankreich und Belgien geflohen. 50 Personen, die in Cadiz zustiegen, waren keine Flüchtlinge, sondern Juden, die in Spanien gelebt hatten und nun die Gelegenheit zur Auswanderung nach Palästina nutzten, um der Diskriminierung von Juden im katholischen Spanien zu entgehen. Obwohl das Franco-Regime den dauerhaften Aufenthalt von Juden im Land zu dieser Zeit nicht gerne sah, gab es Lichtensteins Bericht zufolge große Probleme, für sie Ausreisevisa zu erlangen. Im Oktober 1944 folgte ein zweiter Transport von 425 Flüchtlingen aus den beiden iberischen Staaten. Nun aber war mit der Befreiung Frankreichs das ursprüngliche Ziel, in Spanien Platz für neue Flüchtlinge zu schaffen, der Nachkriegsemigration nach Palästina gewichen. ${ }^{72}$

Der amerikanische Kontinent war ein weiteres mögliches Ziel der Flüchtlinge. Blickenstaffs Büro half bei der Antragstellung bei den Konsulaten. Die größte Gruppe nahm Kanada auf: im Frühjahr 1944 reisten 220 Flüchtlinge dahin aus. Die Zahl derjenigen, die in die USA, Lateinamerika und Großbritannien einreisen durften, lag Wyman zufolge 1944 unter $100 .^{73}$

Deutlich wird, dass die Alliierten trotz der Erkenntnis, dass die Evakuierung der «Altflüchtlinge» aus Spanien dringend sei, lange brauchten, um der Ankündigung Taten folgen zu lassen. Für die spanische Regierung war dies argumentativ von Vorteil. So konnte sie bei Kritik aus dem Ausland darauf verweisen, dass die Alliierten Spanien nur unzureichend die ökonomische

7 Ministerio de Asuntos Exteriores, Oficina de la Información Diplomática: España y los Judios. Madrid 1949, S. 28. Die Äußerung wird hier auf den 18. Januar 1944 datiert. Diese Publikation hatte das Ziel, Spanien als Beschützer der Juden während des Krieges darzustellen. Sie enthält an mehreren Stellen Berichte, die Spaniens Haltung in diesem Sinne verfälschten. Vgl. dazu Rother, Bernd: Franco als Retter der Juden? Zur Entstehung einer Legende. In: Zeitschrift für Geschichtswissenschaft 45 (1997), S. 122-146, S. 141f. Daher kann nicht mit letzter Sicherheit davon ausgegangen werden, dass die Äußerung des Botschafters korrekt wiedergegeben wurde. Sie veranlasste die spanische Seite zu folgendem Kommentar: «Eine Sache ist, mit einem Weltkrieg den Antisemitismus von Hitler und anderen zu bekämpfen, eine andere, gänzlich verschiedene, ist, die Einreise von Juden nach Palästina zu gestatten, auch wenn deren Ablehnung den Tod der 〈Beschützten〉 bedeutete.» Ministerio de Asuntos Exteriores, España, 1949, S. 29.

${ }^{72}$ Leshem; Avni, Spain, S. $116 \mathrm{f}$.

73 Wyman, S. 254. 
Last der Flüchtlinge abnähmen. ${ }^{74}$ Der Plan des War Refugee Board ${ }^{75}$, Spanien zur Drehscheibe der Fluchtrouten zu machen, indem man der Regierung die prompte Wiederausreise garantierte, und diese dadurch zu einer öffentlichen Erklärung, alle Flüchtlinge aufzunehmen, zu bewegen, scheiterte schon im eigenen Hause, an den Widerständen in der US-Administration. ${ }^{76}$

Unterstützung der Flüchtlinge durch Privatpersonen oder durch nichtstaatliche spanische Organisationen ist mit Ausnahme des Roten Kreuzes nicht überliefert. Der wichtigste Grund dürfte sein, dass es nach dem Bürgerkrieg unter der Diktatur eine unabhängig von der Regierung agierende Zivilgesellschaft nur rudimentär gab, nimmt man die Katholische Kirche aus.

\section{Bilanz}

So positiv es zu bewerten ist, dass Spanien in den allermeisten Fällen die Flucht nicht durch Zurïckweisung an der Grenze behinderte und dies auch für Juden galt, so muss doch hervorgehoben werden, dass die wesentlichen Gründe nicht humanitäre Erwägungen oder Ablehnung der nationalsozialistischen Politik waren, sondern die Aufnahmebereitschaft Portugals. Wenn nicht Portugal - seinerseits in der Erwartung baldiger Ausreise nach Übersee - den Transit der Flüchtlinge akzeptiert hätte, wäre auch Spaniens Politik anders ausgefallen. Denn: den Verbleib der politisch in der Regel unliebsamen Flüchtlinge oder ein Anwachsen der kleinen jüdischen Gemeinden lehnte die Regierung ab. Durch die geographische Lage mit einer «offenen» Grenze nach Portugal im Rücken konnte die spanische Regierung ganz anders operieren als Schweden oder die Schweiz, wo die Einreise von Flüchtlingen in aller Regel gleichbedeutend war mit deren Aufenthalt im Land bis Kriegsende.

Über die Zahl der Flüchtlinge, die Spanien im Zweiten Weltkrieg passierten, und die der Juden unter ihnen gibt es sehr voneinander abweichende Schätzungen. Zur Mühlen hält die Angaben der Hilfsorganisationen für den Portugal-Transit, der fast völlig identisch war mit dem Spanien-Transit, für realitätsnah; er geht daher bei insgesamt über 80.000 Flüchtlingen von circa 70.000 Juden aus. ${ }^{77}$ Antonio Marquina plädiert für sehr niedrige Zahlen, ohne sich auf bestimmte Quantitäten festzulegen. Die Angaben der jüdischen

74 Vgl. als Nachkriegsstimme: Ministerio de Asuntos Exteriores, España, 1949, S. 28f.

75 Das WRB war im Januar 1944 von Präsident Roosevelt gebildet worden. Seine Aufgabe war die Rettung von Personen, die von Deutschland verfolgt wurden und sich in unmittelbarer Lebensgefahr befanden. Vgl. zum WRB Wyman, S. 234ff.

76 Vgl. dazu Wyman, S. 250-254.

77 Zur Mühlen, S. 152. 
Hilfsorganisationen aus der Kriegs- und der unmittelbaren Nachkriegszeit hält er für übertrieben, da es für so viele Flüchtlinge überhaupt keine Transportmöglichkeiten von Portugal in Aufnahmeländer gegeben und die größte der Hilfsorganisationen, der JOINT, während des ganzen Jahres 1941 in Portugal über keine effiziente Organisation verfügt habe. ${ }^{78}$ Avni erscheint die von ihm selbst früher gegebene Größenordnung von 30.000 Juden im legalen Transfer zwischen Juni 1940 und Oktober 1942 mittlerweile zu hoch. Die Zahl der Juden, die zwischen Sommer 1942 und der Befreiung Frankreichs zwei Jahre später illegal das Land passierten, veranschlagte er in frühen Publikationen auf $7.500 .^{79}$ Emilienne Eychenne, die sich intensiv mit dem illegalen Grenzübertritt über die Pyrenäen beschäfigt hat, kommt nach einer Auswertung der französischen Archive und nach Zeitzeugenbefragungen zu dem Ergebnis, dass zwischen 15.000 und 50.000 Flüchtlinge illegal Spanien erreicht haben könnten. Genauer ließe sich dies nicht bestimmen. Sie hält aber die niedrigere der beiden Zahlen für realistischer. Den Anteil der Juden schätzte sie 1983 auf nur $10 \%, 1985$ ermittelte sie in einer Stichprobe $20 \%$ Juden. Als Extremwerte wären demnach zwischen 1.500 und 10.000 geflohene Juden vorstellbar, doch entspräche eine Zahl von 3.000 (20\% von 15.000) wohl am ehesten Eychennes Erkenntnissen. Zu ihnen kann man, so Eychenne, weitere 5.000 hinzufügen, die im Sommer 1940 die Grenze passierten. ${ }^{80}$ Was hier fehlt, sind die Juden, die durch die Tätigkeit von Flüchtlingshilfsorganisationen bis Ende 1942 Vichy-Frankreich legal Richtung Spanien verlassen konnten.

Die neueste und umfangreichste Studie zur Flucht aus Frankreich nach Spanien zwischen 1940 und 1944 hat Robert Belot vorgelegt. Sein Augenmerk richtet sich aber fast ausschließlich auf französische Staatsbürger. Zur Zahl ausländischer bzw. staatenloser Juden, die legal oder illegal diesen Weg wählten, äußert er sich nur am Rande. Demnach lag sie bei etwa 20.000; er ergänzt jedoch: «La question mériterait une étude spécifique.» Dieser Zahl wären die Juden mit französischer Staatsbürgerschaft hinzuzufügen, über die er jedoch keine gesonderten Angaben macht. ${ }^{81}$

Solange die Archive der portugiesischen und der spanischen Grenzbehörden nicht systematisch ausgewertet sind, verbleiben uns nur Spekulatio-

${ }^{78}$ Marquina, Política, S. 248f.

79 Avni, Haim: The Spanish-Speaking World and the Jews. The last Half-Century. In: Wistrich, Robert S. (Hg.): Terms of Survival. The Jewish world since 1945. London 1995, S. 358-382, S. 379, Anm. 2; Avni, Spain, S. 91f.; Avni, España, S. $265 f$.

${ }^{80}$ Eychenne, Emilienne: Les Pyrénées de la Liberté. Évasions par l'Espagne, 1939 1945. Paris 1983, S. 252, 323-328. Die Stichprobe in Eychenne, Portes, S. 280.

${ }^{81}$ Belot, Robert: Aux frontières de la liberté. Vichy - Madrid - Alger - Londres. S'évader de France sous l'Occupation. Paris 1998, S. 679, 681, 703 Anm. 22, 751 Anm. 55 (hier das Zitat). 
nen. So könnte man, Avnis neueren Schätzungen folgend, 20.000 legal über Spanien geflohene Juden vermuten. Hinzu kämen, entsprechend Eychennes Ergebnissen, 3.000 Juden, die illegal die Grenze überschritten. Die Gesamtzahl 23.000 erweckt aber einen Anschein von Genauigkeit, der nicht der Realität entspricht. Wir sollten die Schätzung daher noch weiter abschwächen und sagen, dass vielleicht 20.000-35.000 Juden dadurch gerettet wurden, dass Spanien trotz seiner Freundschaft mit Deutschland sie nicht an der Grenze zurückwies. 